\title{
THE IKINEMATICS OF THE SWING PHASE OBTAINED FROM ACCELEROMETER AND GYROSCOPE MEASUREMENTS
}

\author{
Andreas Heyn ${ }^{1}$, Ruth E. Mayagoitia ${ }^{1}$, Anand V. Nene ${ }^{2}$, Peter H.Veltink ${ }^{1}$ \\ ${ }^{1}$ Biomedical Technology Institute, Department of Electrical Engineering, University of Twente, \\ PO Box 217, 7500 AE Enschede, The Netherlands. hill@servidor.unam.mx; p.h.veltink@el.utwente.nl \\ ${ }^{2}$ Roessingh Research and Development, Roessingh Rehabilitation Insitute, Roessinghsbleekweg 33 , \\ 7522 AH Enschede, The Netherlands
}

\begin{abstract}
The kinematics needed to calculate the knee moment during the initial swing phase were obtained from a set of eight leg-mounted uni-axial accelerometers and two gyroscopes. The angular and linear accelerations of shank and thigh were calculated from the signals of two accelerometers mounted on each of the leg segments directed tangentially and radially to the movement. The angular velocities of shank and thigh were measured by the gyroscopes. The absolute angles of shank and thigh were obtained by integration of the gyroscope signal plus an added offset angle, estimated from radial and tangential accelerometer signals registered while standing. Movement was assumed to be in the saggital plane.

The accuracy of the quantities found from the Ieg mounted sensors was calculated in terms of correlation and the RMS error by comparing against measurements obtained by a V][CON ${ }^{\mathrm{TM}}$ system. The results were indistinguishable. The system was later applied in research measurements.
\end{abstract}

\section{INTRODUCTION}

The initial swing phase of gait occurs between $60 \%$ and $73 \%$ of the gait cycle. Since there is no contact with the floor, to obtain the total net moment around the knee $\mathrm{M}_{\mathrm{ok}}$, using the inverse dynamics approach, equation 1 must be solved:

$$
\mathrm{M}_{\mathrm{ok}}=\mathrm{I}_{\mathrm{ol}} \dot{\omega}_{1}+\mathrm{m}_{1} \mathrm{a}_{\mathrm{k}} \mathrm{l}_{\mathrm{cl}}+\mathrm{m}_{1} \mathrm{gl} \mathrm{l}_{\mathrm{cl}} \sin \varphi_{1}
$$

To do this, the angular acceleration of the shank $\dot{\omega}_{1}$, the linear acceleration of the knee $a_{k}^{t}$ and the absolute angle of the shank with the floor $\varphi_{1}$ must be found. The moment of inertia $I_{o l}$, the mass $m_{1}$ and the distance to the center of mass of the shank $1_{\mathrm{cl}}$ can be obtained from tables [1].

Optical movement analysis systems that track markers are very good at finding linear and angular displacements. But given the large amount of noise introduced by double numerical differenciation, accelerations, as given by systems such as the Vicon ${ }^{T M}$, an alternate method for obtaining these values was developed.

\section{METHODS}

Four pairs of uni-axial accelerometers were mounted on two alumnimium strips. The strips were placed anteriorly on the left thigh and shank of young male volunteers. The eight accelerometers measured the tangential and radial accelerations at points $i$ and $i+1$ of each segment (figure 1). The distances between the sensors and the proximal joint (hip or knee) were measured with a measuring tape. A gyroscope was attached to the midpoint of each aluminium strip to measure the angular velocity. Data were recorded at a sampling frequency of $100 \mathrm{~Hz}$.

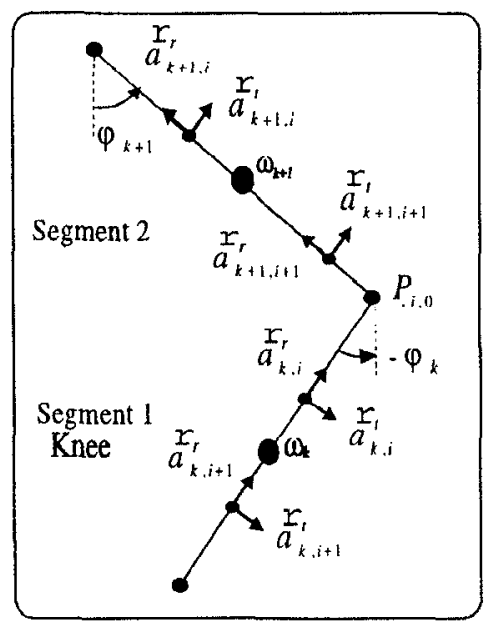

Figure 1. Positioning of the accelerometers and gyroscopes on the thigh and shank

Calibration recordings were made before and after each subject to estimate gains and offsets for all the sensors used.

An optical motion measurement system (Vicon ${ }^{\mathrm{TM}}$ ) was used simultaneously to also obtain the kinematics of the leg segments in the saggital plane. Reflecting markers were placed at the centers of rotation of the left ankle, knee and hip. The position of the markers was tracked by the Vicon ${ }^{T M}$ system using a sampling frequency of $50 \mathrm{~Hz}$.

From the data of the two measurement systems, the absolute angle, the angular velocity, and the angular 
acceleration of the shank, and the linear acceleration of the knee were calculated. The formulas for the calculations based on the leg mounted sensors are in [2].

To detect the different phases of the gait cycle, foot switches under the left heel and the left big toe were used.

\section{RESULTS}

The data in each of the figures are single trials that were recorded simultaneously by both systems, figure 2 while walking at normal speed; figures 3 and 4 while walking fast.

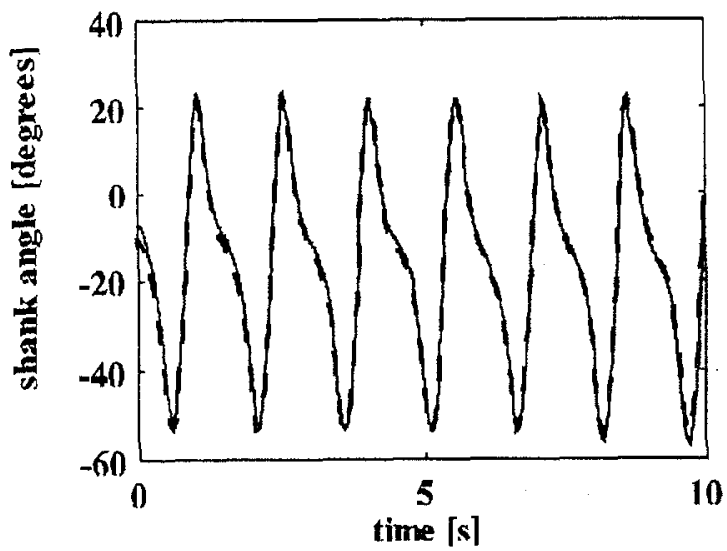

Figure 2. Absolute angle of shank. Optical markers, solid line; first integral of gyroscope, dashed line

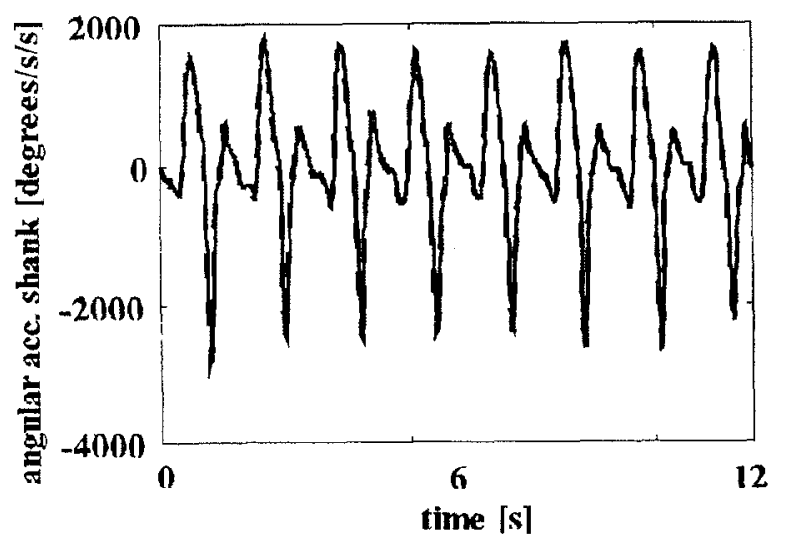

Figure 3. Angular acceleration of the shank. Accelerometer setup, solid line; first derivative of gyroscope, dashed line

Figure 2 shows a typical result of the absolute angle of the shank. The curves obtained from the gyroscopes and from the Vicon ${ }^{\mathrm{TM}}$ system are so close (correlation coefficient = 0.995 , RMS error $=0.026$, normalized $\mathrm{rms}$ error $=0.054$ ) that they can hardly be told appart.

The curves in figure 3 are again very close (correlation coefficient $=0.987$, RMS error $=2.23$, normalized $\mathrm{rms}=0.142$ ). Both were obtained from the leg mounted sensors.
In order to make the comparison with the angular acceleration given by the optical markers (figure 4) it was necessary to process the raw marker data, as the accelerations as given by the Vicon ${ }^{\mathrm{TM}}$ system were not usable. They were treated in the exact same way as the leg mounted sensors. Again the results of comparing the two are very close (correlation coefficient $=0.9889$, RMS error $=128.5228$, normalized $\mathrm{rms}=0.1564$ ).

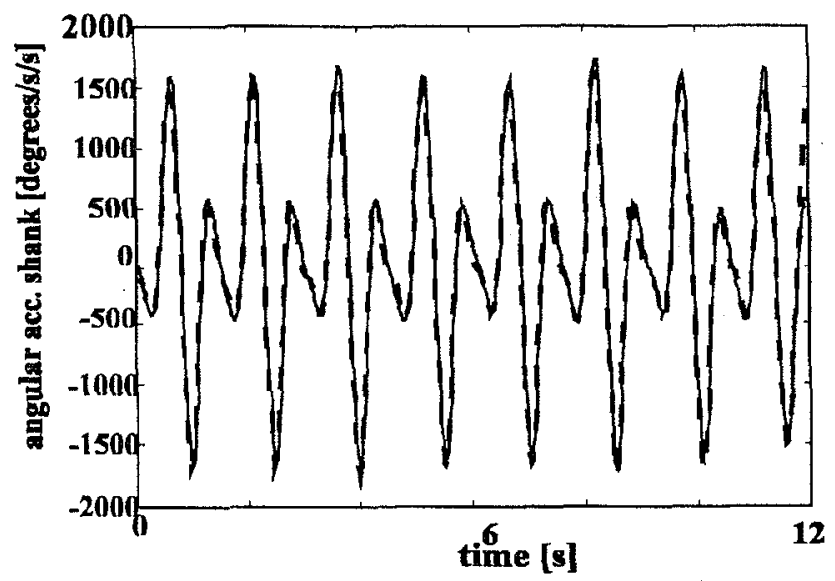

Figure 4. Angular acceleration of the shank. Accelerometer set up, solid line; optical markers, dashed line

\section{DISCUSSION}

The leg mounted sensors give an accurate representation of all the angles, velocities and accelerations and allow the accurate calculation of the net moment around the knee during the swing phase. Thus, this system was used for swing phase measurements, described elsewhere [3].

Some of the calculations needed to extract the quantities from the sensor data are quite intensive. So the full legmounted system is only suitable for off-line applications.

Given the system's portability and relative low cost, it can be applied in any environment. The strips where the sensors are mounted are more cumbersome than the optical markers, but do not impede movement and allow otherwise impossible measurements to be made.

\section{REFERENCES}

[1] B F Le Veau, Williams and Lissner's Biomechanics of Human Motion. Philadelphia, Pa: WB Saunders Company, 3rd edition, 1991.

[2] A T M Willemsen, C Frigo, H B K Boom, "Lower extremity angle measurement with accelerometers --error and sensitivity analysis," IEEE Trans Biomed Eng, vol 38, 1991.

[3] A Heyn, A Nene, R E Mayagoitia: "Activity evaluation of the rectus femoris muscle during the initial swing phase of gait". 11 th ISEK Congress, 1996, submitted. 